\title{
An Insight into Histopathologic Examination as a Gold Standard for the Diagnosis of Chronic Apical Periodontitis
}

\author{
Monica Monea ${ }^{1}$, Anca Maria Pop ${ }^{2}$, Veronica Grozescu³, Alexandra Stoica1, Simona Mocanu4, Cosmin \\ Moldovan ${ }^{5}$ \\ 1. Department of Odontology and Oral Pathology, Faculty of Dental Medicine, University of Medicine and Pharmacy of Tirgu Mures, Romania \\ 2. University of Medicine and Pharmacy of Tirgu Mures, Romania \\ 3. Faculty of Dental Medicine, University of Medicine and Pharmacy of Tirgu Mures, Romania \\ 4. Emergency County Hospital Mures, Tîrgu Mures, Romania \\ 5. Department of Applied Simulation in Medicine, Faculty of Medicine, University of Medicine and Pharmacy of Tirgu Mures, Romania
}

Objective: The purpose of our study was to determine the level of correlation between histopathologic results after surgery for chronic apical periodontitis and the radiographic and clinical diagnosis. The status of gold standard technique of histologic examination was evaluated in the diagnosis of apical radiolucency in necrotic teeth.

Methods: Out of 154 patients with incorrect root fillings and apical radiolucency included in an endodontic retreatment protocol, 87 patients (108 teeth) were scheduled for apical surgery at 3-6 months control recall. Clinical and radiographic exams were completed prior to surgery and compared to the histological results of apical biopsies. The collected data were statistically analyzed with the SPSS version 20.0 and the Chi-square test was used to determine the associations between clinical and histologic diagnosis. A value of $p<0.05$ was considered statistically significant.

Results: There was a statistically significant difference between the number of cases diagnosed as granulomas or cysts during clinical and radiological evaluation compared to histologic evaluation of tissue samples, with $40.9 \%$ to $75.9 \%$ and $54.2 \%$ to $16.8 \%$ respectively $(p<0.05)$. Conclusions: The final diagnosis was obtained only after histologic examination of apical tissue samples, which means that the observations made based on radiologic investigations must be confirmed by biopsy.

Keywords: apical periodontitis, granuloma, apical cyst, radiography, apical surgery

Received 19 October 2017 / Accepted 29 December 2017

\section{Introduction}

Chronic apical periodontitis is defined as an inflammatory disease usually caused by microorganisms located inside the root canals of necrotic teeth. Therefore, the main objectives of endodontic treatment are the thorough cleaning of the infected endodontic system and the adequate aseptic control, aiming to create conditions for the final filling of the entire radicular space with a hermetic tridimensional seal. The success rate of the primary endodontic treatment is considered to be between $86 \%-98 \%$ and its failures are generally caused by one of the following biological factors: persistent infection in the apical third of the root canal, development of an extra-radicular infection, over-filling with endodontic materials responsible for foreign body reactions, cystic lesions and healing by scar tissue $[1,2]$. Unsuccessful cases are always scheduled for conservative retreatment but the success rate in these cases is lower, around $80 \%-85 \%$ [3, 4]. If non-surgical retreatment is not feasible, the best choice remains the apical surgery that assures optimum conditions for healing. Diagnosis of chronic apical periodontitis is based on clinical and radiological examination, which represents important steps

* Correspondence to: Anca Maria Pop

E-mail: ancapop98@yahoo.com in determining the best treatment approach and in cases submitted to apical surgery it should always be confirmed by the histopathologic examination of the periapical tissues. Since many years, histology has been considered as the gold standard by which the true diagnosis of different forms of apical pathology can be confirmed and differentiate, contributing to the development of clinical treatment options in endodontics [5-7]. The purpose of our study was to evaluate the importance of histopathologic examination in the diagnosis of chronic apical periodontitis, by comparison with clinical and radiological findings. As imaging techniques digital radiographs will be used in all cases and cone beam computed tomography only for well selected cases, in order to protect the patients from exposure to higher doses of radiation. The null hypothesis to be tested is that there is a good correlation between clinical, radiographic and histologic examinations and an accurate apical diagnosis can be obtained without histologic evaluation of biopsy specimens.

\section{Methods}

A total of 154 patients with chronic apical periodontitis were examined at the Center for Integrated Dental Medicine of the Faculty of Dental Medicine, University of Medicine and Pharmacy Tîrgu Mureș, from October 2015 to 
June 2017 and consecutively enrolled in this study. Prior to any medical procedure, a written consent was obtained and the investigation was conducted based on principles of the Declaration of Helsinki. Based on clinical and radiological examination using digitally captured radiographs, an indication of endodontic retreatment was made in 138 cases that were considered to have an inadequate primary root canal treatment (incorrect length of the endodontic filling, presence of broken instruments in the apical third of the root canal, poor condensation of endodontic sealer, persistence or enlargement of the apical lesions). At 3-months follow-up, we noted an improvement in 46 cases which therefore had been excluded from the study. The cases without apical radiolucency but persistent clinical symptoms or cases with no signs of improvement were scheduled for CBCT examination. The remaining 108 teeth (87 patients) were scheduled for surgical treatment and used for the final examination protocol that included the recording of age, sex, tooth type, lesion size and the result of histologic examination carried out after apical surgery. The indication for apical surgery was given for teeth with persistent apical radiolucency at 3-6 months follow-up examinations based on the following aspects: persistence of clinical symptoms after endodontic retreatment (pain during mastication, swelling of vestibular gingiva, presence of broken instruments in the apical third that impeded a correct treatment, overfilling with sealer or gutta-percha, presence of posts or bridge abutments that showed lack of healing after endodontic retreatment. Each patient was examined by two authors previously calibrated (MM and AS) that used clinical tests and digital dental radiographs in order to determine the diagnosis; these observations were compared with the histological results of apical tissue biopsies. In 83 cases we obtained enough apical tissue in order to conduct a histologic evaluation, according to a protocol that included: fixation in $4 \%$ buffered formalin solution, embedment in paraffin, cutting into 5 micrometers thick sections that were colored with hematoxylin and eosin stain. The specimens were evaluated by two experienced pathologists (SM and CM) and classified into apical granulomas, cysts or abscesses. The collected data were statistically analyzed with the SPSS version 20.0 and the Chi-square test was used to determine the associations between clinical and histologic diagnosis. A value of $\mathrm{p}<0.05$ was considered to be statistically significant.
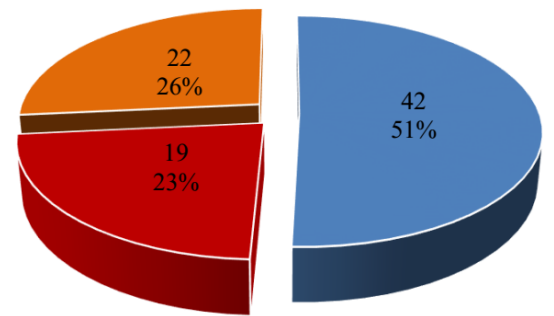

- No signs of inflamation

- Slight discomfort

- Pain on percussion

Fig 1. Distribution of symptoms observed during clinical examination prior to apical surgery

\section{Results}

The mean age of the patients included in this study was $31.4+/-10.5$ years, ranging between $17-58$ years. The distribution of tooth type included $29(34.9 \%)$ upper anterior teeth, $12(14.6 \%)$ upper premolars, $6(7.3 \%)$ upper first molars, $19(22.8 \%)$ mandibular anterior teeth, 14 (16.8\%)
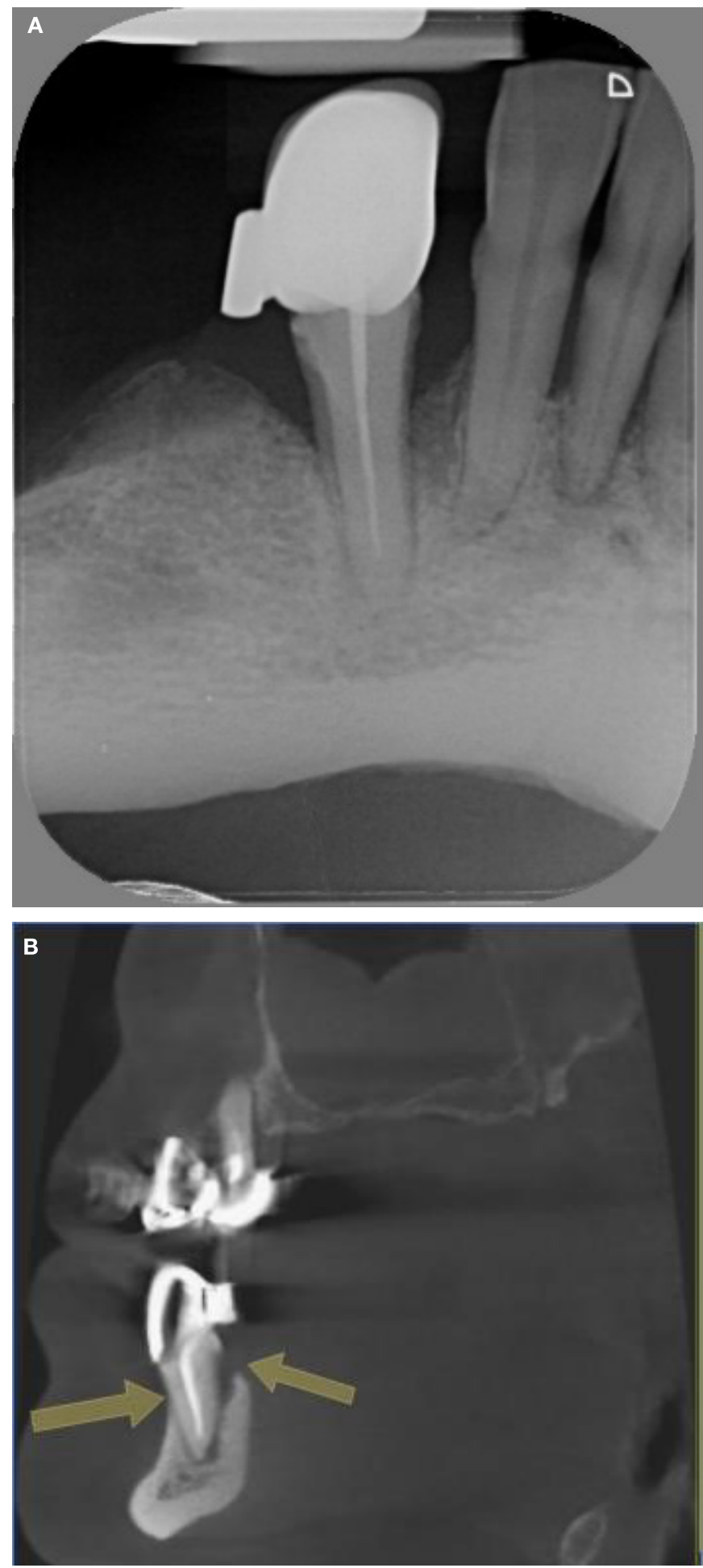

Fig. 2. The radiographic examination after the endodontic retreatment of a lower canine showed an incomplete root filling without apical radiolucency, but the persistent clinical symptoms as slight discomfort during mastication determined us to refer the patient for further investigation. On CBCT the apical and lateral bone defects were evident and the patient was submitted for apical surgery. 
mandibular premolars and $3(3.6 \%)$ lower first molars ( Table I). The majority of affected teeth belonged to the anterior group with a total of 48 cases (57.7\%) followed by the upper first molars. The clinical examination identified no signs of inflammation in 42 cases (50.6\%), slight discomfort during mastication and sensation of tooth egression in 19 cases $(22.8 \%)$ and in 22 cases $(26.6 \%)$ pain on axial percussion was recorded. The results of clinical, radiologic and histopathologic examinations are presented in Table II and Figures 1-4. There was a statistically significant difference between the number of cases diagnosed as granulomas or cysts during clinical and radiological evaluation compared to histologic evaluation of tissue samples, with $40.9 \%$ to $75.9 \%$ and $54.2 \%$ to $16.8 \%$ respectively $(\mathrm{p}<0.05)$. The majority of the cases were diagnosed as cysts (54.2\%) based on clinical and radiographic methods but after histopathologic examination the majority (75.9\%) proved to be granulomas (Table II).

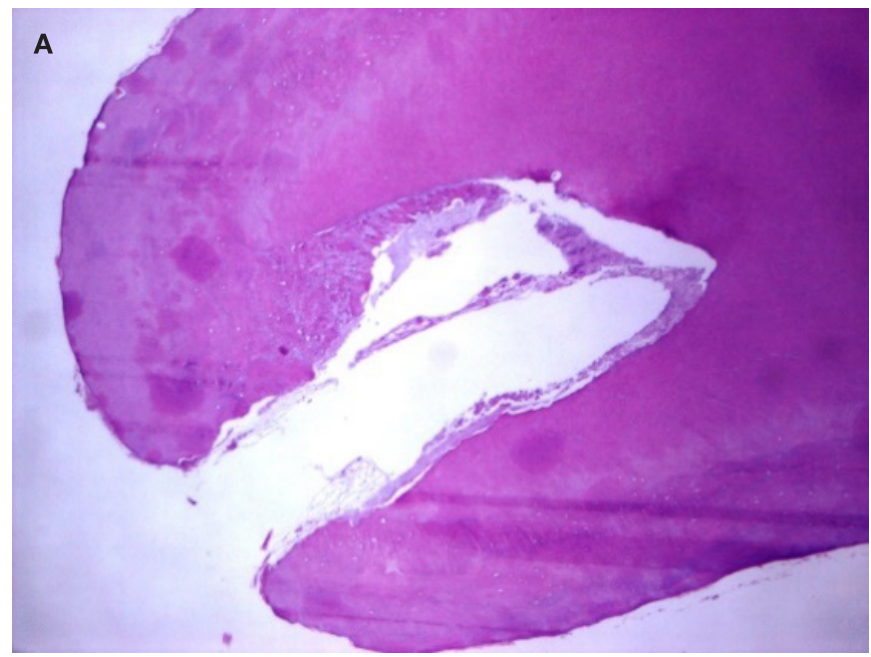

\section{Discussion}

The use of histological samples obtained after apical surgery has a long history in endodontic practice and proved to be

Table I. The distribution of tooth type submitted to apical surgery

\begin{tabular}{lccc}
\hline Upper/lower jaw & Anterior teeth & Premolars & Molars \\
\hline Maxillary teeth & 29 cases $(36 \% 0$ & 12 cases $(15 \%)$ & 7 cases $(9 \%)$ \\
Mandibular teeth & 19 cases $(21 \%)$ & 12 cases $(14 \%)$ & 3 cases $(6 \%)$ \\
\hline
\end{tabular}

Table II. Comparative results after digitally captured radiographs and histopathological examination

\begin{tabular}{lccc}
\hline Diagnosis & Radiographic & Histopathologic & p-value \\
\hline Granuloma & $34(40.9 \%)$ & $63(75.9 \%)$ & $0.026^{\star}$ \\
Cyst & $45(54.2 \%)$ & $14(16.8 \%)$ & $0.001^{\star}$ \\
Abscess & $4(4.81 \%)$ & $6(7.2 \%)$ & 0.398 \\
\hline
\end{tabular}

*statistically significant difference

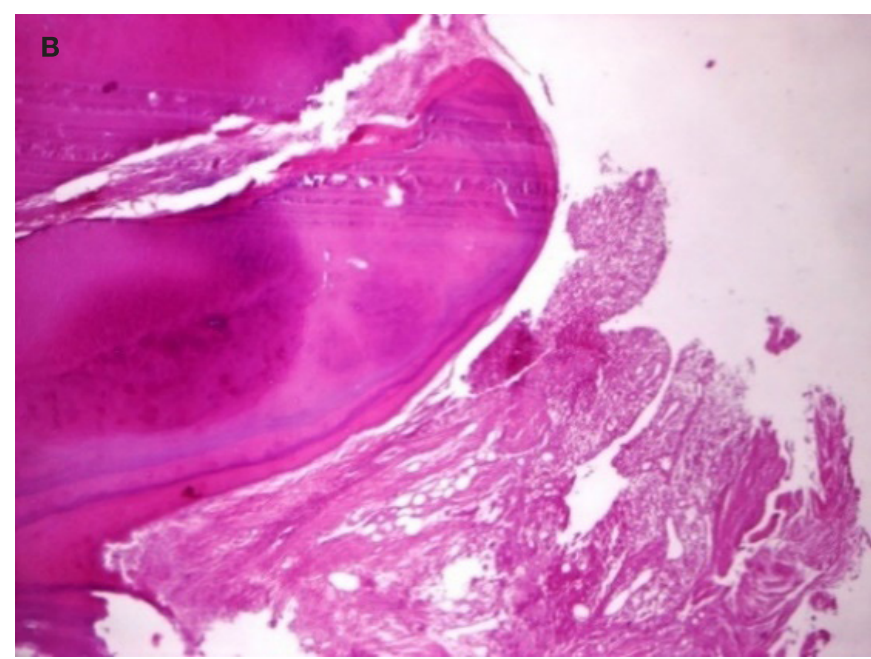

Fig. 3. Histologic specimens obtained from the apical third of the root. A. Apical fragment with radicular dentin, necrotic radicular pulp tissue and inflammatory cells located inside the root canal. B. Granulation tissue attached to the outer surface of the apex and inside the root canal.
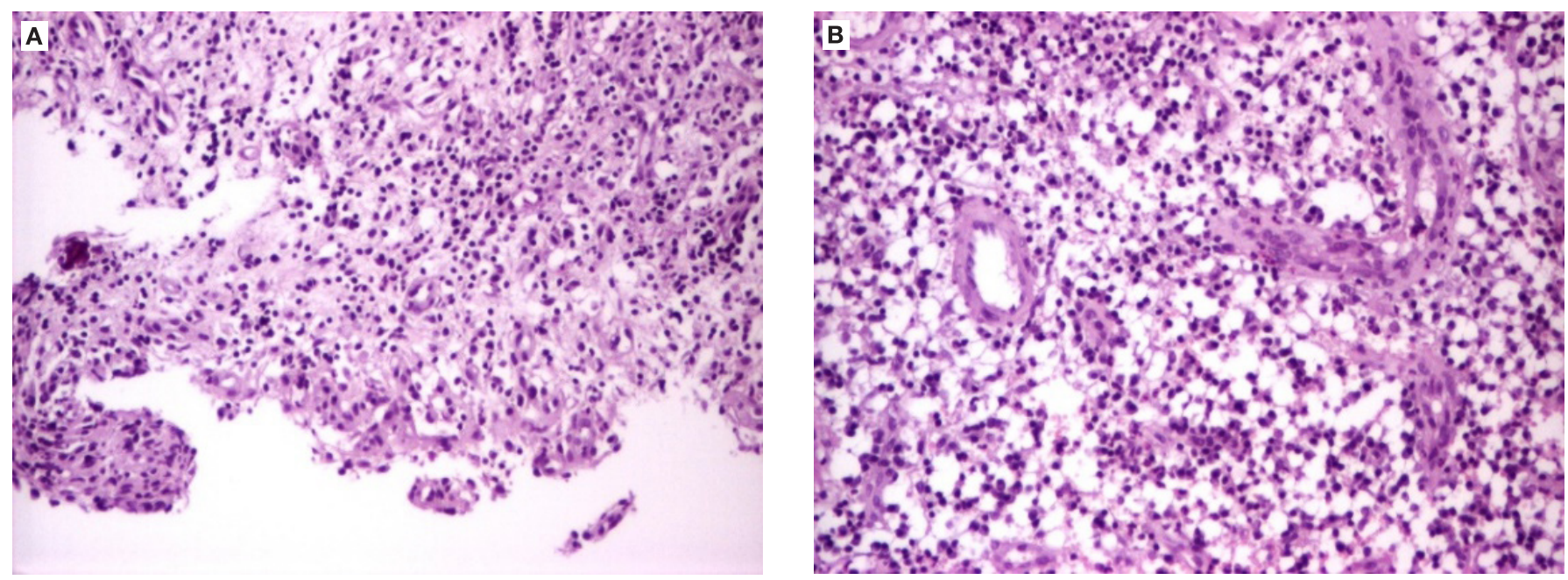

Fig.4. Conjunctive apical granuloma. A. Numerous fibroblasts, lymphocytes and macrophages (HE stain X 10) B. Granulation tissue with rich inflammatory infiltrate and numerous blood vessels, with epithelial islands inside the connecting tissue, representing the indication of a potential apical cyst development. (HE stain, X 10). 
of outmost importance for the development of treatment protocols in cases with chronic apical periodontitis. In our study, we recorded histological results in only $7.3 \%$ and $6.3 \%$ upper and lower molars respectively, which could be attributed to a low submission rate for this type of examination after apical surgery or a preference for extraction in multi-rooted teeth with apical pathology. Diagnosis can be defined as the art of science aiming to distinguish deviations from the state of health; it involves the use of subjective information, clinical tests and imaging techniques. This complex process is carried out also in the case of apical pathology and represents the cornerstone upon which the treatment decisions are based on. Radiographic examination is an essential part in the diagnosis of chronic apical periodontitis; furthermore, it has an important role in the management of endodontic pathology, from treatment plan to the evaluation of therapy outcome. The drawbacks of conventional and digitally captured radiographs may be overcome by using cone beam computed tomography (CBCT) which offers a three-dimensional image of the tooth and its surrounding structures [8-11]. Data from literature confirms that after histologic examination, the majority of cysts diagnosed using conventional radiography proved to be granulomas. In a study conducted by Bornstein et al (2015), the radiographic evaluation of periapical lesions overestimated cystic lesions by $28.4 \%$ compared to CBCT [7]. Patel et al (2012) found that in teeth with primary endodontic disease, the prevalence of apical lesions detected by conventional radiographs and CBCT was $20 \%$ and $48 \%$ respectively [8]. In teeth with irreversible pulpitis, the radiography identified the presence of apical pathology in 3\% and the CBCT in $14 \%$ of the cases [9]. Nevertheless, according to the American Association of Endodontists the CBCT must be considered an adjunct to 2-dimensional imaging techniques reserved only for special cases [12]. Campello et al (2017) found that the accuracy of CBCT was much higher than the digital radiography in detecting artificially created periapical lesions. The correct diagnosis recorded using CBCT was $73.6 \%$ and by radiograph $56.9 \%$ of the cases, the greatest differences being observed in maxillary $(71.4 \%$ and $28.6 \%)$ and multi-rooted teeth $(83.3 \%$ and $33.3 \%)$ respectively [12].

In clinical practice, the digital retroalveolar radiographs are the most common type of investigation used in the case of apical lesions $[9,13]$. It is generally accepted that radiography is not a perfect diagnostic method, as it gives a bi-dimensional image of a three-dimensional structure and furthermore, specific clinical and biological aspects my not be visible on radiographs. Thus, the presence of an apical lesion sometimes is not directly evident and the extension and spatial relationship with anatomical landmarks are not always visualized. With all the limitations of conventional radiographs in showing small bone lesions, a systematic review conducted by Petersson et al [14] concluded that this type of examination has a high capacity in detecting normal apical conditions. In our study the radiographic ex- amination was based on specific criteria in differentiating the cysts from granulomas, as the size of the lesion, degree of radiolucency, aspect of the borders. Accordingly, a radiolucent lesion with irregular periphery and less than $10 \mathrm{~mm}$ diameter was considered granuloma, whereas intense radiolucent defects with well-defined borders and more than $10 \mathrm{~mm}$ diameter were considered cysts.

There were numerous attempts in the scientific literature to distinguish between granulomas and apical cysts based entirely on their radiographic aspect: the granuloma has an indistinct border while cysts are characterized by a visible limit. The presence of a proliferating epithelial tissue without a cystic cavity was considered to have the potential of cystic transformation [15-17]. According to the scientific literature, the incidence of granuloma and cyst is between $9.3 \%-87.1 \%$ and $6 \%-55 \%$ respectively, due to different classification criteria used in histologic studies [17-19]. In the study conducted by Kondori et al (2011) the endodontists were not able to reach the correct diagnosis of apical granulomas in $42.2 \%$ of the cases with apical pathology, compared to $45.9 \%$ error diagnostics made by general dentists. This had probably led to unnecessary surgery, as apical granulomas can be successfully treated by conservative endodontic therapy. Many studies have emphasized the need to subject all periapical lesions removed during surgery to histopathological examination $[5,20]$. The results of our study confirmed as well that histopathology is superior to radiography in the accurate diagnosis of apical granulomas and cysts. Histopathologic examinations that identify other pathologic conditions than granulomas, cysts, abscesses or scar tissue are estimated to represent $0.7 \%-5.0 \%$ of all periapical biopsies [21]. These can be benign aggressive lesions as central giant-cell granuloma, fibro-osseous lesions as periapical cemental dysplasia or many primary and metastatic malignant lesions.

Our histopathologic results showed the high incidence of apical granulomas which is in accordance to other studies $[22,23]$. We found that $75.9 \%$ of specimens were granulomas and $16.8 \%$ were cysts. In $7.2 \%$ of the cases the results showed the presence of apical abscesses, compared to $4.8 \%$ after clinical and radiographic examination, results that were not statistically significant. A better correlation of diagnosis in these cases was determined probably due to specific clinical aspects of the apical abscess: deformation of alveolar bone, intense pain, slightly extruded tooth or mobility. Von Arx et al [24] discussed the prognostic factors of apical surgery, suggesting that the healing rates increase if there is no history of preoperative pain, the size of the lesion is less than $5 \mathrm{~mm}$ wide and the root filling is well condensed. Simon et al [6] compared CBCT images and histological results of periapical biopsies regarding the ability to differentiate apical cysts from granulomas, concluding that $\mathrm{CBCT}$ proved to be clinically more precise and more useful. Furthermore, when used for diagnostic purposes, it was noted that CBCT detects radiolucent changes of the apical bone more often and in initial stages than conven- 
tional radiographs, which allows an earlier endodontic or surgical treatment. CBCT is a powerful tool for endodontic diagnosis, treatment and follow-up but its advantages must be correlated to the higher radiation dose compared to digital radiographs [25, 26]. Dental specialists need an evidence-based methodology based on further clinical studies, regarding the use of $\mathrm{CBCT}$ during endodontic treatment in order to make a correct medical decision on both treatment outcome or need for surgical interventions.

\section{Conclusions}

Periapical lesions represent the most frequent apical pathology diagnosed by conventional radiographic examinations. However, due to the limits of these methods, some cases remain misdiagnosed. The radiographic diagnosis of these osteolytic lesions may be hindered by their structural and evolution variations; the apical root area is difficult to be truly assessed on a two dimensional image and the third dimension information is essential in surgery planning.

Based on the results of our study, the retroalveolar digital radiography was characterized by a limited capacity in detecting periapical lesions when compared to histopathological examinations. Therefore, the null hypothesis was not confirmed as we could not reach the correct diagnosis of the apical lesions based only on clinical and radiographic examination. The poor correlation observed between the histopathologic diagnosis compared to clinical and radiological data led us to the conclusion that the former can be considered the gold standard diagnostic method in chronic apical periodontitis. It is recommended that all chronic apical lesions that did not respond to conservative therapy to be submitted to histopathologic examination, as there is a slight possibility to be the sign of other severe diseases.

In order to reduce unnecessary surgical interventions in teeth with adequate endodontic treatments but persistence of apical radiolucency, the use of advanced imaging techniques as CBCT might be a good option in well selected cases.

\section{Conflict of interest}

None to declare.

\section{Acknowledgement}

This study was conducted under the framework of the research grant no. 3688/01.04.2016, financed by SC COSAMEXT SRL Tîrgu Mureș and developed by the University of Medicine and Pharmacy of Tîrgu Mureș.

\section{References}

1. Nair PN. On the causes of persistent apical periodontitis: a review. Int Endod J 2006; 39: 249-281.

2. Tanalp J, Gungor T. Apical extrusion of debris: a literature review of an inherent occurrence during root canal treatment. Int Endod J 2014; 47: 211-221.

3. De Chevigny C, Dao T, Basrani BR, Marquis V, Farzaneh M, Abitbol S, Friedman S. Treatment outcome in endodontics: the Toronto study Phase-4: initial treatment. J Endod 2008; 34: 258-263.

4. Torabinejad M, Corr R, Handysides R, Shabahang S. Outcomes of nonsurgical retreatment and endodontic surgery: a systematic review. J Endod 2009; 35: 930-937.

5. Ramanpreet B, Simarpreet SV, Rajat B, Amandeep B, Shruti G. Histopathological insight into periapical lesion: An institutional study from Pujab. Int Oral Maxillofac Pathol 2012; 3(3): 2-7.

6. Simon JHS, Enciso R, Malfaz JM, Roges R, Bailey-Perry M, Patel A. Differential diagnosis of large periapical lesions using cone beam computed tomography measurements and biopsy. $J$ of Endodontics. 2006; 32: 833-837.

7. Bornstein MM, Bingisser AC, Reichart PA, Sendi P, Bosshardt DD, Von Arx T. Comparison between radiographic (2-dimensional and 3-dimensional) and histologic findings of periapical lesions treated with apical surgery. J Endod 2015; 41(6): 804-811.

8. Patel S, Wilson R, Dawood A, Mannocci F. The detection of periapical pathosis using periapical radiolucency and cone beam computed tomography - part 1: Pre-operative status. Int Endod J 2012; 45(8): 702-710.

9. Patel S, Durack C, Abella F, Shemesh H, Roig M, Lemberg K. Cone beam computed tomography in Endodontics - a review. Int Endod J 2015; 48: 3-15.

10. Fernandy R, Cadavid D, Zapata SM, Alvarez LG, Restrep FA. Impact of three radiographic methods in the outcome of nonsurgical endodontic treatment: a five-year follow-up. J Endod 2013; 39: 1097-1103.

11. Paula-Silva FWG, Wu M, Leonardo MR, Bezerra da Silva L, Wesselink PR. Accuracy of Periapical Radiography and Cone-Beam Computed Tomography scans in diagnosing apical periodontitis using histopathological findings as a gold standard. J Endod 2009; 35 (7):1009-1012.

12. Campello AF, Goncalves LS, Guedes FR, Marques FV. Cone-beam computed tomography versus digital periapical radiography in the detection of artificially created periapical lesions: A pilot study of the diagnosis accuracy of endodontists using both techniques. Imaging Sci Dent 2017; 47(1): 25-31.

13. Tewary S, Luzzo J, Hartwell G. Endodontic radiography: who is reading the digital radiographs? J Endod 2011; 37(7): 919-921.

14. Petersson A, Axelsson S, Davidson T, Frisk F, Hakeberg M, Kvist T. Radiological diagnosis of periapical bone tissue lesions in endodontics: A systematic review. Int Endod J 2012; 45(9): 783-801.

15. Saraf AP, Kamat S, Puranik RS, Puranik S, Saraf S, Singh BP. Comparative evaluation of immunohistochemistry, histopathology and conventional radiography in differentiating periapical lesions. J Conserv Dent 2014; 17(2): 164-168.

16. Shen J, Zhang H, Gao J, Du X, Chen Y, Han L. Short-term observation of clinical and radiographic results of periapical surgery: a prospective study. Biomedical Research 2016: 27(3): 923-928.

17. Matsuda V, Kadowaki CAN, Kataoka SHN, Caldeira CL. A comparison of clinical, histological and radiographic findings in periapical radiolucid lesions. Dental Press Endod 2011; 1(3): 17-21.

18. Kanagasingam S, Lim CX, Yong CP, Mannocci F, Patel S. Diagnostic accuracy of periapical radiography and cone beam computed tomography in detecting apical periodontitis using histopathological findings as a reference standard. Int Endod J 2017; 50(5): 417-426.

19. Kondori J, Mottin RW, Laskin DM. Accuracy of dentists in the clinical diagnosis of oral lesions. Quintessence Int 2011; 42: 575-577.

20. Goel S, Nagendrarely SG, Raju MS, Krishmojirao DJ, Rostogi R, Mohau RS, Gupta S. Ultrasonography with color Doppler and power Doppler in the diagnosis of periapical lesions. Indian J Radiol Imaging. 2011; 21:279-283.

21. Peters E, Lau M. Histopathologic examination to confirm diagnosis of periapical lesions: A review. J Can Dent Assoc. 2003; 69(9): 568-600.

22. Song M, Jung IY, Lee SJ, Lee CY, Kim E. Prognostic factors for clinical outcomes in Endodontic Microsurgery: A retrospective study. J Endod. 2011; 37: 927-933.

23. Song M, Chung W, Lee SJ, Kim E. Long-term outcome of the cases classified as successes based on short-term follow-up in Endodontic microsurgery. J Endod 2012; 38:1192-1196.

24. Von Arx T, Jensen SS, Hanni S, Friedman S. Five-year longitudinal assessment of the prognosis of apical microsurgery. J Endod 2012; 38:570-579.

25. Leonardi Dutra K, Haas L, Porporatti AL, Flores-Mir C, NascimentoSantos J, Mezzomo LA. Diagnostic accuracy of cone-beam computed tomography and conventional radiography on apical periodontitis: a systematic review and meta-analysis. J Endod 2016; 42: 356-364.

26. Venskutonis T, Plotino G, Tocci L, Gambarini G, Maminskas J, Juodsbaly SG. Periapical and endodontic status scale based on periapical bone lesions and endodontic treatment quality evaluation using cone beam computed tomography. J Endod 2015; 41(2): 190-196. 